\title{
Los clusters industriales y sus implicancias estratégicas: Una visión de América Latina
}

\author{
Vera Garnica, José*
}

\section{Resumen}

Este artículo tiene por finalidad analizar el estado de desarrollo de los clusters industriales en América Latina, y los factores de competitividad como variable estratégica. Para ello se revisan diversos enfoques teóricos que ponen énfasis en aquellos rasgos que generan eficiencia al conjunto de las empresas pertenecientes al cluster, adicionalmente se revisan experiencias de clusters exitosos y competitivos a nivel internacional, identificando los factores de éxito. Se determinó que el mayor desarrollo de los clusters está condicionado por la calidad del ambiente local; por la concentración de habilidades y conocimientos; y en la capacidad de compartir de sus empresas. Muchos de estos atributos están ausentes en los clusters de América Latina, donde sí se observa una gran brecha productiva entre empresas grandes y pequeñas; un bajo nivel de especialización y cooperación, y un bajo nivel de innovación tecnológica. Se concluye que para enfrentar estos desafíos los Gobiernos deberán priorizar una política basada en el reconocimiento de los clusters como ejes de las economías, y realizar una mayor inversión en la generación de conocimientos y desarrollo tecnológico. Por su parte, las empresas deben desarrollar competencias basadas en la especialización y la cooperación.

Palabras clave: Clusters, competitividad, localización.

\section{Industrial Clusters and Their Strategic Implications: A Vision of Latin America}

\begin{abstract}
The purpose of this article is to analyze the state of development of industrial clusters in Latin America, and competitive factors as a strategic variable. Diverse theoretical positions that place emphasis on the aspects that generate efficiency in the businesses that form the clusters are reviewed, and also experiences from successful competitive clusters are analyzed, in order to identify success factors. The study determined that the greatest cluster development was conditioned by the quality of

Recibido: 05-06-12. Aceptado: 05-12-05

Magíster en Administración y Dirección de Empresas. Doctor en Estudios Empresariales, Universidad de Los Lagos. Chile. E-mail: jvera@ulagos.cl
\end{abstract}


the local environment, by the concentration of knowledge and abilities, and by the competitive capacity of the member companies. Many of these attributes are lacking in Latin American clusters, where a great productive gap is observed between large ands small companies, as well as a low level of technological innovation. The conclusion is that in order to confront these challenges, governments must create priorities based on a policy of recognition of clusters as economic axes, and invest larger investments in the generation of technological knowledge and development. On the other hand, the industries must develop competence based on specialization and cooperation.

Key words: Clusters, competitiveness, localization.

\section{Introducción}

Desde la década de los ochenta se viene estudiando con mucha fuerza la conformación y funcionamiento de los clusters industriales (concentración geográfica de empresas interrelacionadas) como factor relevante en el éxito económico de una nación. Su justificación radica en que el análisis de los sistemas económicos va más allá de los agregados tradicionales: la empresa, los sectores de producto o los grandes sectores como son la industria y los servicios, debiendo concentrarse en los clusters porque "estos tienen más que ver con la naturaleza de la competencia y las fuentes de ventaja competitiva" (Porter, 1999a).

Comprender el funcionamiento de los clusters y la forma en que sus integrantes se relacionan adquiere relevancia para las empresas, instituciones de gobierno y la sociedad en su conjunto. Para las empresas constituye un elemento a considerar en sus nuevos desafíos; las relaciones efectivas serán parte de las competencias que una empresa debe poseer para relacionarse con la economía global. Para las instituciones de gobierno el conocimiento de los clusters sirve de orientación para el diseño de políticas públicas que tengan como objetivo el desa- rrollo económico y productivo de ciertas áreas que van más allá del análisis tradicional de sectores industriales. Ello permite, también, establecer un nuevo paradigma en la forma de relacionarse con los integrantes del clusters.

Para las economías en desarrollo, especialmente para los países Latinoamericanos -donde gran parte de los clusters lo componen micro y pequeñas empresas- los desafíos para superar los bajos niveles de innovación tecnológica, de especialización, y cooperación, están dados por un claro conocimiento de los distintos tipos y características de clusters existentes en sus economías y la fase de desarrollo en que estos se encuentran. A partir de ese diagnóstico generar instancias de fomento y desarrollo que tengan como propósito transformar los agrupamientos de empresas en articuladores efectivos del crecimiento de las economías locales.

\section{Antecedentes y experiencias}

Las experiencias y estudios respecto de los clusters se han venido incrementando en la última década debido a las implicancias que su actividad tiene en el desarrollo local. Porter (1999a) destaca las relaciones que se establecen entre los in- 
tegrantes de un cluster para mejorar la productividad, el desarrollo de innovaciones, y la generación de nuevas empresas. Ser parte de un cluster les permite a las empresas operar de un modo más productivo en la obtención de insumos; el acceso a la información, la tecnología y las instituciones necesarias; la coordinación con las compañías relacionadas; y en la forma de encarar y medir las mejoras.

En cuanto a la innovación Porter (1999a) señala que los clusters juegan un papel vital en la capacidad de innovar continuamente de las empresas, dado que los compradores más exigentes suelen formar parte del cluster. Las empresas de computación radicadas en Silicon Valley y en Austin, Texas, se relacionan con los clientes para determinar sus necesidades, eso le permite detectar las tendencias del mercado antes que las empresas radicadas en otras regiones. Paralelamente, las relaciones con otras entidades que integran el cluster ayudan a las empresas a anticiparse a la evolución de la tecnología, la disponibilidad de componentes, y a nuevos conceptos de servicio y marketing.

Los clusters también estimulan el surgimiento de nuevos negocios; proliferan nuevos proveedores porque la existencia de una base concentrada de clientes reduce los riesgos. Los clusters desarrollados incluyen industrias relacionadas que demandan insumos comunes o similares.

Los estudios de Porter (1999a) también están referidos a los múltiples vínculos $\boldsymbol{y}$ sinergias generadas por las empresas participantes del cluster. Destaca como ejemplo el cluster del vino de California; otro ejemplo es el cluster italia- no del cuero, que incluye varias cadenas de industrias relacionadas.

Altenburg (2001) por su parte se refiere a que el especto más interesante de los clusters es su capacidad de crear nuevas ventajas competitivas y estabilizarlas mediante procesos continuos de innovación tecnológica. Lo que define el desempeño competitivo de un cluster no es la cantidad de vinculaciones productivas o acciones conjuntas, sino la dinámica de aprendizaje tecnológico. El análisis de clusters debería por lo tanto enfocar los mecanismos de generar y difundir conocimientos, esto implica estudiar y promover el acceso de clusters a fuentes externas de conocimientos, especialmente en países en vías de desarrollo que no operan a la altura de las últimas tecnologías mundiales.

Maskell (2001) al referirse a los factores que inciden en el crecimiento de los clusters, argumenta que las razones están en el desarrollo de los sistemas de innovación. Indica que es la interdependencia o ajuste entre la "estructura económica" del cluster y las "instituciones" la que posibilita ese crecimiento. De cualquier manera, eso se reflejará en que las ventajas (economías de aglomeración y menores costos de transacción) superan a los inconvenientes (congestión y sobresaturación de los mercados), prácticamente inexistente en un principio.

Otros estudios donde se destaca la importancia de las interacciones, están referidos a la política de cluster. Los análisis cluster han puesto de manifiesto la importancia y necesidad de tomar en consideración una serie de factores que estaban prácticamente ausentes en el análisis tradicional. La importancia del territorio y de las externalidades que genera la 
concentración geográfica de actividades; la importancia de las interacciones y de la cooperación, que trascienden los límites sectoriales tradicionales y que con frecuencia no están basadas en relaciones de mercado; la idea de que las empresas y sectores no se pueden ver aisladamente, sino que forman parte de un sistema que condiciona su modo de funcionamiento y resultados; el hecho de que en ese sistema las restantes organizaciones e instituciones juegan un significativo papel. Todo ello ha hecho que se planteen nuevos fundamentos y, ligado a ellos, nuevas respuestas de la política industrial (Almqvist et al., 1998).

En este nuevo enfoque, la política de clusters, que sustituye a las tradicionales políticas sectoriales, se convierte en la "piedra angular de la política industrial en muchos países" (Roelandt y Den Hertog, 1998). Se propugna favorecer la cooperación e interacción, pero sin menoscabo del clima competitivo. Se piensa que el gobierno puede actuar como catalizador e intermediario, así como coordinador o incluso autoridad, en los clusters, ayudando a superar los bloqueos y desequilibrios organizacionales e institucionales y conflictos que impiden el buen funcionamiento del cluster.

\section{Desafíos para la gestión empresarial}

Los factores presentes hoy en el mercado conducen a un nuevo paradigma en la gestión empresarial. Hoy, además de ser eficiente, hay que conseguir enfocarse de manera extraordinaria hacia el cliente y, en muchas ocasiones, hacia el consumidor final.
A fin de superar las limitaciones de la planificación estratégica convencional, varias empresas líderes vienen experimentando en torno a lo que llaman competencias o capacidades nucleares. En lugar de formular la pregunta "¿en qué negocio estamos realmente?”, realizan un sondeo más profundo, considerando "qué aptitudes y conocimientos técnicos especiales que aportamos a la empresa en la que estamos ahora pueden servir como base para erigir empresas futuras".

Esta no son ideas nuevas, Selznik (1957) definió muchas de ellas en la década de 1950. En esta misma línea Prahalad (1999) al estudiar los desafíos emergentes que debe enfrentar la empresa se refiere que esta "debe ir más allá de la calidad y rapidez de sus productos y servicios que prestan, atributos si bien son necesarios no son suficientes para competir con éxito. Tendrán que sumarles la capacidad para reconocer las principales discontinuidades emergentes globalidad, desregulación, volatilidad, convergencia, fronteras industriales difusas, normas, desintermediación y sensibilidad ecológica- y aprender a ser innovadoras. Las discontinuidades convierten algunas competencias centrales en peligrosas rigideces, por lo tanto, a fin de explotar las oportunidades que irán surgiendo, están obligadas a desarrollar nuevas competencias, tienen que enfrentar la tarea simultánea de "olvidar" ciertas cosas, y de aprender otras".

Una de las tareas que deberá encarar la empresa, principalmente aquella perteneciente a economía en desarrollo, es la formación de alianzas temporarias: asociaciones y acuerdos de cooperación destinados a transferir habilidades y co- 
nocimientos entre empresas, generando la Capacidad para compartir. El espacio más adecuado para generar dicha capacidad puede provenir de la pertenencia a clusters industriales.

\section{Fundamentos teóricos de los clusters}

No existe precisión acerca del nacimiento de la teoría de complejos productivos, si bien es cierto que ya Marshall (1920) estudió los factores externos en la ubicación de industrias especializadas. La geografía económica era conocida, desde hace mucho tiempo, como un campo importante en una extensa literatura, sobre todo en los primeros 50 años del siglo XIX. Sin embargo, con la economía neoclásica, la economía geográfica perdió vigencia. En los últimos años, debido al surgimiento de nuevas teorías sobre el crecimiento económico y el comercio internacional, aumentó el interés en el campo de la geografía económica y con ello, la teoría de complejos productivos fue ganando espacios.

Es posible separar los diferentes trabajos e investigaciones empíricas en dos grupos claramente diferenciados. Por un lado la corriente principal de la economía (neoclásica), salvo algunas excepciones, ha ignorado el desarrollo de los clusters. Si bien en algunos escritos de economistas clásicos (Marshall, 1920, por ejemplo), hay argumentos relacionados con el tema, y existen en la actualidad desarrollo recientes (Krugman, 1991, 1995, 1998) que los han revitalizado, en general esta corriente no ha prestado atención a la conexión entre complejos productivos y crecimiento.
Por otro lado, se ha desarrollado otra corriente que ha nacido justamente intentando explicar la experiencia observada en los distritos industriales italianos y aglomeraciones de empresas en otras partes del mundo.

\subsection{Los economistas clásicos y los clusters}

Existen dos enfoques básicos para comprender los beneficios de la concentración: la perspectiva marshalliana (Marshall, 1920), que toma como punto de partida las economías de escala externas y su presencia en "distritos industriales"; y las teorías de localización industrial que se construyeron sobre los aportes de Weber (1929) y Hoover (1937), donde los beneficios se denominan economías de aglomeración.

Marshall (1920) definió a las economías de escala externas como el ahorro de costos de las empresas producido por el tamaño o crecimiento del producto de la industria. Tales economías contrastan directamente con las economías de escala internas, que son la fuente de los rendimientos crecientes, producto del aumento en el tamaño de planta. Tales economías externas son esencialmente economías espaciales, que pueden ser definidas generalmente como efectos económicos producidos por la proximidad entre actores económicos. Estos pueden ser tanto positivo como negativos, estático como dinámicos, pecuniarios o tecnológicos.

Weber (1929) identificó economías de aglomeración -definidas como el ahorro de costos que experimentan las firmas como su resultado de incrementar la con- 
centración espacial- como una de las tres causas primarias de la concentración espacial o la aglomeración. Por su parte Hoover introdujo una distinción entre urbanización y economías de localización.

En la literatura sobre cluster, el eje es principalmente sobre las externalidades (Marshall, 1920 y Schmitz, 1995, 1997) relacionadas con la proximidad entre las empresas (economías de localización), diferente a las externalidades asociadas con las ventajas urbanas generales (economías de urbanización).

\subsection{Los rendimientos crecientes y la competencia imperfecta}

De acuerdo a los principios básicos de la teoría neoclásica, con mercados competitivos, homogeneidad de productos y rendimientos a escala no crecientes, no hay razones para preocuparse desde la política pública por un mayor encadenamiento productivo entre actividades económicas. En estos casos la economía se moverá a lo largo de su frontera de producción en respuesta a las señales de precios internacionales y todas las actividades con potencial para incrementar el ingreso nacional se organizarán en el sistema. De esta manera las actividades productivas se localizarán en las regiones donde se encuentren los insumos básicos, o cerca de los centros de consumo, o en zonas aleatorias, dependiendo de factores como costos de transporte del insumo principal versus el producto final, o la disponibilidad de otros recursos adicionales en la región (manos de obra, capital).

Por el contrario los modelos más recientes, tanto de la nueva geografía económica como los de la nueva teoría del crecimiento, se basan fundamentalmente en la existencia de economía de escala a nivel de cada empresa. Es decir, en el reconocimiento que para diversas industrias, no es lo mismo producir una determinada cantidad en diversas plantas dispersas espacialmente, que realizar una producción conjunta en un solo lugar. En ausencia de tales economías, como la teoría neoclásica postula, los productores no tendrían ningún incentivo a concentrar sus actividades y, en consecuencia, abastecerían a los consumidores desde varias plantas locales. Ahora bien, la presencia de economías de escala a nivel de las empresas, que persisten en un tramo de producción bastante importante, socava los supuestos de competencia perfecta (principalmente la existencia de muchos oferentes), ya que en estos casos sobrevivirán pocas empresas produciendo estos bienes. Por lo tanto surge la necesidad de utilizar modelos de competencia imperfecta.

4.2.1. Teoría del crecimiento y los clusters: La aglomeración sectorial

En los aportes de las nuevas teorías del crecimiento está la clave para explicar el desarrollo de los clusters, estén o no basados en recursos naturales. En primer lugar, estas nuevas teorías han demostrado la importancia de la acumulación de cierto recurso específico para explicar el crecimiento económico.

Por un lado, a nivel teórico, hay fuertes evidencias (Lucas, 1988) en el sentido de que una vez acumulada una cierta masa crítica de capital humano éste presenta fuertes externalidades $y$, en particu- 
lar, se constituye en el principal factor de la atracción o expulsión de trabajadores y de capital físico de una determinada región.

Por otro lado, a nivel empírico, los estudios que han explicado la formación y desarrollo de algún cluster en particular, han resaltado la importancia del papel que ha jugado la formación de un pool de capital humano específico a las actividades propias de la región. Por ejemplo, en su estudio de dos clusters dinámicos en América Latina (lácteos en Argentina y fruta fresca en Chile), Casaburi (1999) destaca varios factores que influyeron en la competitividad que estos desarrollaron. En primer lugar, se confirma la importancia de la presencia de una gran cantidad de productores medianos con energía empresarial. También se destaca la existencia de un sistema científico tecnológico eficaz y especializado en el recurso de la región.

Aunque el capital humano, en última instancia, es específico para cada persona o para cada empresa, es evidente que buena parte del conocimiento científico y tecnológico es de naturaleza "no rival" (el consumo por parte de un individuo no impide que esas mismas cantidades sean consumidas por otros), como también lo han destacado las nuevas teorías del crecimiento, de tal modo que esos conocimientos tienen, en alguna medida, las características de un bien público.

A partir de esta cualidad, la teoría del crecimiento permite entender por qué les resulta conveniente agruparse, o al menos interactuar, a las empresas o a las personas que comparten una cierta dotación de recurso específico, en lugar de aislarse y competir: es a partir de esta in- teracción que ellas pueden apropiarse de las externalidades del capital humano de otros.

4.2.2. Nueva geografía económica y Clusters: La aglomeración regional

La nueva geografía económica se concentra en explicar la estructura espacial de la economía a través de ciertos artificios técnicos para producir modelos en los cuales hay rendimientos crecientes y mercados de competencia imperfecta (Krugman, 1998). Estos artificios comprenden:

1. El modelo de competencia monopolística, que al asumir un continuo de bienes, permite respetar la naturaleza entera de las decisiones localizacionales y analizar el modelo en términos del comportamiento de variables continuas como la participación industrial en una región particular.

2. El concepto de iceberg, que destaca el hecho de que en la teoría de la localización, los costos de transporte son esenciales. Específicamente el concepto hace alusión a que una fracción del bien transportado se pierde (es decir, se "derrite") en el tránsito, a una tasa constante por unidad de distancia.

3. El concepto de evolución incluido en los modelos de la Nueva Geografía Económica, que implican usualmente equilibrios múltiples. Asumen un proceso de ajuste ad hoc, en el cual los factores de producción se mueven gradualmente hacia localizaciones que ofrecen retornos reales más elevados. De esta manera las localizaciones son estratégicas.

4. La tecnología y la computación debido, fundamentalmente, a que inclu- 
Los clusters industriales y sus implicancias estratégicas: Una visión de América Latina Vera Garnica, José

so los modelos más simples de geografía económica son muy complejos, por lo cual su solución requiere tanto cálculos estáticos como simulaciones dinámicas, que exceden las posibilidades del cálculo normal.

En buena medida, la nueva geografía económica se desarrolló, persiguiendo como objetivo suplir las falencias que presentaban los modelos tradicionales de comercio, bajo contextos de competencia imperfecta, rendimientos crecientes a escala y costos de transacción. Sus principales exponentes argumentan que si bien los modelos tradicionales de comercio, que contemplan los supuestos mencionados, han mostrado los efectos de la integración económica sobre la competitividad relativa de los países desarrollados, o industriales, y los países en desarrollo, pueden mencionarse al menos tres falencias importantes en dichos modelos (Puga, 1998).

En primer lugar, los modelos de comercio explican las divergencias en estructuras productivas solo a través de diferencias en las dotaciones de factores. Comienzan suponiendo que hay países "grandes" y países "pequeños", sin brindar una explicación del origen de esas diferencias ni mencionando por qué países que inicialmente eran similares, luego desarrollan estructuras productivas diferentes.

En segundo término, tampoco explican por qué las empresas de ciertos sectores tienden a localizarse cerca unas de otras, conduciendo así a una especialización regional.

Por último, asumen que el desarrollo industrial se produce gradualmente y simultáneamente en todos los países, cuando la evidencia empírica muestra que los procesos de industrialización se producen en forma de olas en las que las industrias se distribuyen de países en países.

En respuesta a estas falencias, la nueva geografía económica formaliza desarrollos basándose en la tendencia de las empresas y los trabajadores a radicarse en las proximidades de los mercados grandes con el propósito de mostrar qué regiones similares inicialmente, pueden luego diferenciarse a través de mecanismos endógenos que las lleven a constituir regiones centrales y periféricas.

La mayor parte de los modelos de la nueva geografía económica postulan que la concentración geográfica de las actividades económicas surge de la interacción entre rendimientos crecientes, costos de transporte y demanda. Las formalizaciones más importantes de esta teoría han surgido a partir de tres modelos básicos: Krugman y Venables (1993), Krugman (1991) y Venables (1996).

Los encadenamientos verticales

(Venables, 1993), constituyen una de las razones de la endogeneidad del tamaño del mercado a diferentes localizaciones. El desplazamiento espacial de la industria ubicada en la fase avanzada de la cadena productiva afecta el mercado de la industria que se sitúa en una etapa previa de la misma. En este contexto ¿cuáles son las fuerzas que conducen a la aglomeración de la industria en una única localización? Es posible aseverar que los incentivos a la aglomeración proceden del carácter vertical de la relación interindustrial. Por una parte, existe un eslabo- 
namiento de demanda, dado que las empresas en la industria que se encuentran en una fase previa de la cadena productiva se benefician de la proximidad de las empresas que le compran los insumos. Por otra parte, existe un eslabonamiento de costos, ya que las empresas de la industria que se encuentran en la fase posterior de la cadena productiva se benefician de la cercanía de los oferentes. Dado que ambas industrias son imperfectamente competitivas, las empresas de las mismas desearán ubicarse mutuamente cerca y ella genera potencialmente aglomeraciones.

\subsection{El modelo de la ventaja competitiva de las naciones}

Los estudios de la ventaja competitiva de las naciones de Porter (1990) y los determinantes de dicha ventaja nacional señalan que la competitividad de una nación depende de la capacidad de su industria para innovar y mejorar. La ventaja competitiva se crea y mantiene a través de un proceso muy localizado en el que influyen características muy propias de una nación, como sus valores, cultura, estructura económica, instituciones e historia.

La diversidad e intensidad de las relaciones funcionales entre empresas explican la formación de un complejo productivo y su grado de madurez. Estas relaciones se refieren a los cuatro puntos de lo que él denomina "rombo de la competitividad", es decir, de las relaciones de apoyo, con productores de insumos complementarios y con proveedores de insumos y factores especializados, y que en definitiva constituyen las fuentes de la ventaja competitiva. Al operar de forma simultánea en el tiempo y en el espacio, estos aspectos crean las condiciones para la formación y el desarrollo de los clusters en determinados lugares.

Los clusters constituyen uno de los vértices del rombo de la competitividad (sectores afines y auxiliares), pero es mejor verlos como una manifestación de las relaciones existentes entre los cuatro vértices. La forma como se manifiestan esas fuentes de competitividad y como interactúan entre sí, permite explicar cómo hacen las empresas para generar, mantener, o perder sus ventajas competitivas. Al poner la atención en estos aspectos, se está reconociendo que las empresas no existen en una especie de vacío social, sino que operan en entornos geográficos, económicos, sociales y culturales específicos, y que el análisis de sus estrategias de competitividad actuales o potenciales, debe considerar ciertas características esenciales de esos entornos, para que tenga un verdadero poder explicativo.

Es por ello que las categorías de análisis de las empresas individuales, como pueden ser las cuatro vías para el aumento de la productividad, no bastan para entender cómo fue que una empresa determinada desarrolló su competitividad. Antes, es preciso analizar las condiciones de la competitividad que existen en el clima de negocios de la empresa, un clima de negocios que suele estar estructurado por complejas redes de relaciones entre empresas y organizaciones públicas y privadas. Los cuatro aspectos que se destacan permiten encontrar y comprender los determinantes esenciales de la competitividad, dentro de la multiplici- 
Los clusters industriales y sus implicancias estratégicas: Una visión de América Latina Vera Garnica, José

dad de relaciones, actores y causas que actúan en el clima de negocios.

\subsection{Los distritos industriales y eficiencia colectiva}

Schmitz (1995) argumenta que los aportes de los autores clásicos y los desarrollos más recientes dentro de la corriente económica principal son insuficientes para explicar el nacimiento y expansión que han experimentado varios complejos productivos alrededor del mundo. De allí, que surgen conceptos como "economías externas" y "acción conjunta" que ayudan a explicar y a comprender el funcionamiento de los clusters.

El concepto de "economías externas" es importante para comprender las ganancias de eficiencia que las empresas pueden derivar de su participación en un cluster. El problema, sin embargo, es que dicho concepto está restringido a ganancias o pérdidas no planificadas. El hecho esencial del concepto de efecto externo es que el efecto producido no es una creación deliberada sino un subproducto accidental, no deliberado de alguna actividad legítima.

Por lo tanto, estos efectos accidentales son de gran importancia en el desarrollo de los complejos productivos contemporáneos. Estos efectos son también producto de acciones y decisiones conscientes por parte de los agentes económicos. Esto último se conoce como "acción conjunta".

Para tener en cuenta estos dos aspectos, Schmitz (1990; 1995) desarrolló el concepto de "eficiencia colectiva" para describir las ventajas competitivas derivadas de las economías externas y la acción conjunta.

La acción conjunta es un elemento crítico para comprender el crecimiento y la competitividad de los clusters, y está estrechamente relacionada con la noción de cooperación interempresas producto de la confianza y el capital social (Humphrey y Schmitz, 1995; Nadvi, 1997; 1999).

Una identidad sociocultural común es propensa a favorecer códigos de comportamiento que inducen la confianza y la cooperación y a crear sanciones sociales. La confianza, sin embargo, no surge sólo de la identidad cultural, los valores compartidos y las normas de comportamiento que gobiernan las relaciones interempresas pueden emerger de relaciones puramente comerciales.

Tal acción conjunta puede ser de dos tipos, cooperación de empresas individuales y grupos de empresas que juntas fuerzas en asociaciones comerciales, consorcios productivos, etc.

Conceptualmente las economías externas existen cuando los costos o beneficios privados (es decir percibidos por un agente económico en particular) difieren de los sociales (es decir, los que percibe la sociedad en su conjunto). Cuando los costos sociales son más altos que los costos privados, se habla de deseconomías externas (principalmente en el estudio de los efectos de la contaminación ambiental); mientras que cuando los beneficios sociales son más altos que los privados se hace referencia a las economías externas (que es lo que interesa en el análisis de cluster). 


\section{Los clusters y sus implicancias estratégicas}

El mapa económico del mundo actual está dominado por lo que Porter (1999a) denomina los "clusters": masas críticas -ubicadas en determinado lugarde inusual éxito competitivo en determinados campos. Agrupan a una amplia gama de industrias y otras entidades relacionadas que son importantes para competir. Incluyen, por ejemplo, a proveedores de insumos críticos -como componentes, maquinarias y servicios -, $y$ a proveedores de infraestructura especializada. Con frecuencia también se extienden aguas abajo -hasta canales y clientes- $y$, lateralmente, hasta fabricantes de productos complementarios y empresas que operan en industrias relacionadas por sus habilidades, tecnologías o insumos comunes. En muchas ocasiones los clusters incluyen organismos gubernamentales y otras instituciones- universidades, agencias encargadas de fijar normas, centros de estudios, proveedores de capacitación y asociaciones de comercio- que proveen entrenamiento, educación, información, investigación y apoyo técnico.

Los clusters representan una nueva clase de organización espacial, que puede catalogarse como un punto intermedio entre los mercados aleatorios, por un lado, y las jerarquías, o integraciones verticales, por el otro. Un cluster, por lo tanto, es una manera alternativa de organizar la cadena de valor. Si se le compara con las transacciones entre compradores y vendedores dispersos, se advierte que la proximidad entre empresas e instituciones localizadas en determinado lugar -y los repetidos intercambios entre ellas- alientan una mejor coordinación y mayor confianza. Por consiguiente, los clusters mitigan los problemas inherentes a las relaciones aleatorias, sin imponerles la inflexibilidad de la integración vertical ni plantear los desafíos implícitos en la creación y preservación de vínculos formales, como redes, alianzas y asociaciones. Los clusters afectan a la forma de competir aumentando la productividad de las empresas radicadas en la zona; imponiendo el rumbo y el ritmo de la innovación; y estimulando la formación de nuevas empresas.

Para la OECD (1999) los clusters son "redes de producción de empresas fuertemente interdependientes (incluyendo proveedores especializados), ligadas unas a otras en una cadena de producción que añade valor; así pues, "el concepto cluster va más allá de las redes horizontales simples, en las cuales, las empresas que operan en el mismo mercado de productos finales y pertenecen al mismo grupo industrial cooperan en ciertas áreas" e igualmente en otro lugar, "el análisis de clusters va más allá del análisis sectorial tradicional, ya que toma en consideración los lazos con firmas que se encuentran fuera de los límites sectoriales tradicionales". La OECD agrega que "en algunos casos, los cluster también comprenden alianzas estratégicas con universidades, institutos de investigación, servicios empresariales intensivos en conocimiento, instituciones puente (comisionistas, consultores) y clientes".

\section{El ámbito local en la competitividad}

No sólo las discontinuidades señaladas por Prahalad (1999) influyen en el 
desempeño de las empresas, el ámbito nacional de un país influye en la competitividad de las empresas localizadas dentro de esa nación. El contexto nacional es importante debido a que en muchas industrias el mercado se ha convertido en un espacio global, donde empresas de diversos países compiten de igual a igual en todo el mundo. En tales mercados globales, para algunas firmas es muy fácil obtener el éxito debido a que están localizadas en países que poseen ventajas competitivas en ciertas industrias.

Cuando la competitividad estaba muy atada al costo de los insumos, los lugares con alguna característica clave -disponibilidad de recursos naturales, o mano de obra barata- disfrutaban de una ventaja comparativa que era perdurable y decisiva desde el punto de vista competitivo.

En la economía actual, la competitividad es mucho más dinámica. Las empresas pueden mitigar muchas de las desventajas derivadas del costo de los insumos que necesitan mediante la provisión global, convirtiendo la antigua noción de la ventaja comparativa en un factor menos relevante. Hoy, la ventaja competitiva depende de un uso más productivo de los insumos, lo cual exige permanente innovación. Sin embargo, todas las industrias pueden utilizar tecnología de avanzada, y todas pueden hacer uso intensivo del conocimiento. El factor diferenciador radica en la calidad del ambiente local de negocios y la forma en que las empresas compiten en ese lugar geográfico (Porter, 1999b).

"En una era de competencia global, la geografía económica plantea una paradoja. Cabría esperar que la localización perdiera importancia, en virtud de medios de transporte rápidos, comunicaciones de alta velocidad y fácil acceso a los mercados. Pero ocurre exactamente lo opuesto. Las ventajas competitivas duraderas suelen ser locales, y surgen de la concentración de habilidades y conocimiento, instituciones, empresas rivales, negocios relacionados y clientes exigentes. La proximidad geográfica y una cultura similar generan relaciones más estrechas, mejor información, mayores incentivos, y otras ventajas derivadas de la productividad y la innovación que son difíciles de explotar a la distancia. $Y$ cuanto más compleja, dinámica y basada en el conocimiento se vuelve la economía mundial, más cierto es este principio" (Porter, 1999b).

Conocer el ámbito nacional conlleva examinar la estructura competitiva industrial, como también la etapa de desarrollo industrial. Examinar el ambiente industrial significa evaluar el impacto de la globalización en la competencia dentro de una industria y si facilita el logro de ventajas competitivas en el mercado mundial (Hill y Jones, 1996).

Kanter (2003) plantea que en el futuro el éxito será para aquellas empresas, grandes y pequeñas, que sean capaces de satisfacer los criterios globales y utilizar las redes globales, y corresponderá a aquellas ciudades, estados y regiones que mejor realicen la tarea de conectar a las empresas que operen en ellas con la economía global. El poder no procede de la ubicación en sí, sino de la capacidad de dominar uno de los activos intangibles que hacen que los clientes sean fieles. Estos activos son los conceptos (ideas, diseños o formulaciones de vanguardia 
para productos o servicios que crean valor para los clientes), la competencia (capacidad de convertir las ideas en aplicaciones para los clientes, de ponerlas en práctica al más alto nivel de exigencia), y las conexiones (alianzas entre empresas con el fin de aprovechar las capacidades esenciales, crear más valor para los clientes o simplemente abrir las puertas y ampliar los horizontes).

\section{Los clusters en América Latina}

Resulta evidente que las circunstancias de desarrollo económico de los países de América Latina distan mucho de las economías avanzadas, son muchos los indicadores que se pueden señalar, en el plano empresarial el nivel de productividad impulsado por los procesos de innovación y desarrollo tecnológico parece ser lo más evidente. Esta realidad se manifiesta en forma clara en la formación, desarrollo y competitividad de los clusters de América Latina.

Porter (1999a) señala al respecto que los clusters de los países en vías de desarrollo suelen tener menos profundidad y amplitud; que necesitan componentes, servicios y tecnologías extranjeros; que las empresas competitivas funcionan más como islas que como integrantes de un cluster; que los clusters suelen tener menos miembros que en los países desarrollados y las redes en que operan son más bien radiales jerárquicas en torno a unas pocas empresas de grandes dimensiones; que la comunicación es escasa y las relaciones entre empresas e instituciones mal desarrolladas; que la formación de clusters se ve dificultada por la escasa formación y capacitación de la mano de obra, las carencias tecnológicas, la falta de acceso al capital, el insuficiente desarrollo de las instituciones y una política estatal inadecuada (ya que restringe el asentamiento, protege de la competencia, no ajusta los programas de universidades y escuelas técnicas a las necesidades de los clusters...).

América Latina presenta una gran variedad de aglomeraciones de empresas con distintas características en cuanto a número y tamaño, competitividad, y capacidad innovadora. Algunos clusters están cambiando rápidamente por la propia dinámica de la economía (liberalización económica, apertura comercial, configuración de bloques comerciales, entre otros) lo que conlleva, en algunos casos, a la pérdida de competitividad de muchas actividades sustitutivas de importaciones.

Según Altenburg (2001), en América Latina se pueden distinguir en forma clara cinco tipos de clusters: Clusters de Micro y Pequeñas Empresas; Clusters de Empresas productoras de bienes de consumo; Clusters basados en el procesamiento de recursos naturales; Clusters de Empresas de servicios intensivos en conocimientos; y Clusters dominados por empresas transnacionales.

Gran parte de los clusters existentes en América Latina se componen exclusivamente de micro y pequeñas empresas en actividades caracterizadas por barreras de entrada muy bajas en términos de capital y conocimientos. El grado de especialización en ese tipo de empresas es muy bajo, y generalmente incluyen pocos eslabones de la cadena de valor; el nivel de acción conjunta suele ser bajo comparado con clusters de empresas 
medianas en el sector formal, debido a la falta de confianza para cooperar en beneficio mutuo; la capacidad innovadora es muy limitada.

Los clusters medianamente diversificados de empresas productoras de bienes de consumo, se caracterizan por agrupar diferentes estratos de empresas, abarcando desde microempresas hasta grandes y medianas empresas con altos niveles de eficiencia técnica y gerencial. La mayoría se han especializado en bienes de consumo masivo (vestuario, calzado, muebles), pero también en productos con un nivel de tecnificación un poco más alto (productos eléctricos, cerámica). Además, producen a escalas mayores y con bienes de capital mucho más modernos. Sin embargo, el nivel de innovación y especialmente las inversiones en I\&D también suelen ser mínimas, dado que las empresas se dedican casi exclusivamente a actividades maduras basadas en maquinaria importada y procesos estandarizados.

El tercer tipo de clusters está referido a las empresas basadas en el procesamiento de recursos naturales. Este grupo es bastante heterogéneo en términos de sectores, tamaño e importancia económica. Incluye clusters que surgieron en torno a sectores agropecuarios (productos lácteos, carne, frutas), forestales (industria de celulosa) y mineros (industria petroquímica y metalúrgica). Algunos de estos clusters se desarrollan en torno a una o varias empresas muy grandes que se han diversificado, desarrollando una amplia gama de actividades manufactureras y de servicios ligados a la explotación primaria. A partir de los años 90 la privatización de empresas y la libe- ralización de los regímenes de inversión han favorecido procesos de terciarización (outsourcing), alianzas estratégicas, co-inversiones, fusiones y adquisiciones, con el resultado de modificación en estructuras empresariales más diferenciadas que pueden profundizar el grado de especialización interna de los clusters.

Los clusters de empresas de servicios intensivos en conocimientos constituyen una cantidad más bien reducida. Un fenómeno relativamente tradicional son los centros bancarios que se han desarrollado en Panamá y algunas islas caribeñas, así como las Zonas Francas que se fundaron en Manaus (Brasil) y ciudad Colón (Panamá), entre otros. Estos clusters deben su existencia a una legislación especial que otorga incentivos a inversionistas. Aunque básicamente albergan empresas de una actividad principal -offshore banking en el caso de los centros financieros mencionados, importación y exportación en las Zonas Francas también constituyen una especie de clusters, dado que se están desarrollando servicios conexos. Un fenómeno más reciente es los clusters de empresas de software en Montevideo (Uruguay) y el Valle Central de Costa Rica.

En los últimos años se ha venido desarrollando otro tipo de clusters de empresas transnacionales que operan en sectores de alto contenido tecnológico, como la industria automotriz y electrónica. Estos clusters surgen en el marco de estrategias de globalización de grandes consorcios transnacionales y se concentran en Brasil y México. La participación de empresas nacionales queda limitada al rol de proveedores de insumos sencillos e indirectos. De ahí resulta un rasgo 
típico de los clusters transnacionales: aunque tengan un nivel tecnológico alto y existan interacciones entre diferentes eslabones de la cadena de valor, el grado de vinculación con el empresariado nacional y el potencial para procesos de aprendizaje a nivel del clusters son bajos.

Altenburg (2001) identifica tres rasgos comunes que dificultan el desarrollo de los clusters en América Latina. En primer lugar, la estructura empresarial se caracteriza por una gran brecha productiva entre empresas pequeñas y grandes. Según cifras de la Comisión Económica para América Latina y el Caribe (CEPAL) la productividad laboral de las empresas micro y pequeñas de la región es aproximadamente 10 veces más baja que aquella de las empresas medianas y grandes. Además, la productividad laboral de las primeras se ha estancado por varias décadas, por lo cual la brecha productiva se está ensanchando continuamente- (Weller, 2000). La gran mayoría de las micro y pequeñas empresas puede clasificarse como "sobrevivientes" informales o semi-formales que carecen de visión estratégica, excelencia técnica y capital, y por lo tanto se han desacoplado del progreso tecnológico.

El bajo nivel de especialización y cooperación es otro de los rasgos que presentan los clusters de la región si se compara con los clusters innovadores en Norteamérica y Europa. Son escasas las empresas que producen bienes de capital y las que ofrecen servicios especializados e intensivos en conocimientos.

La especialización recíproca está más desarrollada entre las empresas modernas de mediano y gran tamaño. Debido a la gran brecha productiva así como a deficiencias en términos de calidad, precio y fiabilidad de entrega, estas empresas compran muy pocos insumos y servicios a micro y pequeñas empresas. Así el potencial para arrastrar a micro y pequeñas empresas es limitado y se profundiza la heterogeneidad estructural. A diferencia de los clusters "Porterianos", las empresas grandes no se desempeñan como articuladores de un sistema productivo integrado; no corresponden al modelo del "developmental entreprise" que terciariza servicios e impulsa la especialización y con eso (voluntariamente o no) va abriendo nuevos nichos de mercado y creando oportunidades de aprendizaje tecnológico en su entorno empresarial.

En lo que se refiere a la cooperación, los empresarios latinoamericanos muestran escasa disposición a tomar acciones conjuntas para mejorar su posición competitiva. Los estudios de Altenburg concluyen que la falta de cooperación no sólo se observa en la relación entre las empresas, sino también en la relación entre empresas e instituciones de fomento.

Una tercera limitante está referida a la baja innovación tecnológica aplicada por las empresas. Estas se desempeñan en actividades de bajo contenido tecnológico, por lo tanto no dedican muchos recursos a la investigación y desarrollo de nuevos productos. Las instituciones públicas de investigación y enseñanza, además de la falta de recursos, a menudo carecen de una clara orientación empresarial (Sutz, 2000).

El bajo nivel tecnológico no es sólo un problema de los clusters, sino un rasgo general de la estructura productiva de la región. Esto encuentra justificación en la baja inversión en I\&D que realizan los países de América Latina y el Caribe. 
El grado de desarrollo y madurez de los cluster de América Latina aún es incipiente. Los estudios de Ramos (1998) sobre los complejos productivos en torno a los recursos naturales señalan que la expansión de las actividades ha estado concentrada en las fases iniciales de procesamiento a diferencia de los grandes complejos productivos de los países desarrollados que ya han alcanzado un nivel de madurez significativo. Ramos agrega que se necesita de una estrategia para avanzar hacia la elaboración de productos especiales y más sofisticados con mayor valor agregado nacional, es decir, diseñar procesos que aceleren las múltiples actividades que tienden a aglomerarse en torno a los recursos naturales, sin contradecir las tendencias naturales del mercado y potenciando los encadenamientos con actividades proveedoras de insumos, equipos e ingeniería (hacia atrás), así como los encadenamientos con actividades procesadoras y usuarios de los recursos naturales (hacia adelante). De lo que se trata es de lograr que los complejos productivos incipientes en torno a la abundante base de recursos naturales de la región puedan convertirse con más rapidez en complejos maduros.

La evolución típica que cabría esperar en el desarrollo y conformación de un complejo productivo maduro exitoso, es decir, uno capaz de mantener su competitividad, no sólo por su ventaja comparativa natural, sino cada vez más en virtud de mejoras continuas de la productividad, es la que señala Ramos (1998): En la primera etapa sólo se extrae y exporta el recurso natural, importándose todo lo necesario para su producción. El procesamiento local es mínimo. En una segunda etapa se sustituyen algunos insumos y quizás aumenta el nivel de procesamiento antes de exportarlo. Se comienza a sustituir importaciones con producción local de algunos insumos y de equipos (típicamente bajo licencia para el mercado nacional). En la tercera etapa, ciertos insumos, que se fabrican localmente y que surgieron para sustituir importaciones, suben en la curva de aprendizaje y se comienzan a exportar y, al mismo tiempo, aumentan la cantidad y el valor de los productos procesados localmente en el mix de exportaciones de la región.

Finalmente, en la cuarta etapa, el total de actividades del cluster es competitivo en los mercados mundiales $y$, además de exportar, las empresas locales comienzan a invertir en otras regiones con dotaciones similares de factores.

\section{Conclusiones}

Las nuevas formas de organización industrial basada en los clusters que escapan a los tradicionales sectores industriales- implica que las empresas e instituciones se interconectan y relacionan en forma sistémica en torno a una actividad productiva principal. Ello constituye una tendencia mundial, eso sí, con distintos matices sea que se trate del nivel de desarrollo de los países y de sus economías, del nivel tecnológico y de innovación, o del grado de desarrollo y madurez de la actividad productiva o de negocios. Este nuevo escenario internacional caracterizado por el desarrollo de los agrupamientos industriales o clusters sumado a una profundización de la globalización imponen verdaderos desafíos a las empresas en su forma de gestionar y vincularse entre ellas y sus stakeholders. 
La experiencia internacional está atiborrada de ejemplos exitosos en cuanto a los beneficios logrados por los clusters. Pero es cierto también que el mayor desarrollo está precisamente en aquellos clusters de economías más avanzadas en desarrollo tecnológico e innovación, especialización, transferencia y aplicación de conocimientos. Este panorama dista bastante de la realidad latinoamericana siendo atribuido el menor desarrollo de los clusters precisamente a la falta de aquellos factores que constituyen la fortaleza de los países desarrollados. El desafío está entonces en priorizar una política por parte de los gobiernos basada en el reconocimiento de los clusters como eje de las economías, y una mayor inversión en la generación de conocimiento y desarrollo tecnológico.

En coexistencia a los procesos de globalización encontramos que los entornos locales y de sus instituciones juegan un papel importante en el desarrollo de la capacidad creativa e innovadora de las empresas y de los clusters. La economía global y el ambiente local no son términos antagónicos, por el contrario, ambos procesos actúan como factores sinérgicos, precisamente la globalización debe su fuerza a la complejidad del conocimiento, existencia de factores competitivos y a una dinámica empresarial territorial.

La empresa - principalmente aquella perteneciente a economías en desarrollo como unidad económica central de los clusters, debe reconocer este nuevo escenario y adquirir aquellas competencias centrales generadoras de ventaja competitiva. La capacidad para generar acuerdos de cooperación y la capacidad para compartir deben ser algunas de ellas.
En lo que respecta a los clusters de América Latina sus tareas pendientes están referidas a acortar la brecha productiva entre empresas pequeñas y grandes, aumentar los niveles de especialización y cooperación en las pequeñas empresas y trabajar decididamente por la innovación tecnológica.

\section{Referencias Bibliográficas}

Altenburg, Tilman (2001). "La promoción de clusters en América Latina", Publicaciones FOCOPymes, Bs. Aires.

Almqvist, Gunilla, Norgren, Lennart y Strandell, Anne-Christine (1998). "Clusters and Cluster Policy in Sweden". NUTEK, The Swedish National Board for Industrial and Technical Development.

Casaburi, Gabriel G. (1999). "Dynamic Agroindustrial Clusters: The political Economy of Competitive Sectors in Argentina and Chile", First published in the United State, St. Martin's Press, Inc.

Hill, Charles, y Jones, Gareth (1996). "Administración Estratégica. Un enfoque integrado". Mc Graw Hill, tercera edición.

Hoover, Edgar M. (1937). "Spatial price discrimination". Review of Economic Studies, 4.

Humphrey, John. y Schmitz, Hubert (1995). "Principles for promoting clusters and networks of SMEs", United Nations Industrial Organization (UNIDO).

Kanter, Rosabeth M. (2003). "Prosperar localmente en la economía global", Harvard Deusto Business Review, № 119.

Krugman, Paul (1991). "Increasing returns and economics geography", Journal of Political Economy, 106.

Krugman, Paul (1995)."Development, Geography, and Economic Theory". Cambridge-Massachusetts: The MIT Press.

Krugman, Paul (1998)."What's new about the new economic geography?". Oxford Review of Economic Policy 14 (2). 
Los clusters industriales y sus implicancias estratégicas: Una visión de América Latina Vera Garnica, José

Krugman, Paul y Venables Anthony (1993). "Integration, specialization and adjustment". European Economic Review. Vol. 40.

Lucas, Robert E. Jr. (1988). "On the mechanics of Economics Development", Journal of Monetary Economics, January 3-42.

Marshall, Alfred (1920). "Principles of Economics", 8th Edition, London. Macmillan.

Maskell, Peter (2001). "Growth and the territorial configuration of economic activity" Dirección en internet, http/wwwdruid.dk/ conferences/nw/paper1/maskell.pdf Paper to be presented on the DRUID Summer Conference, June 12-15.

Nadvi, Khalid M. (1997). "The cutting edge: collective efficiency and international compettitiveness in Pakistan", IDS Discussion Paper 364, Institute of Development Studies, Sussex.

Nadvi, Khalid M. (1999). "Facing the new competition asociations in devolping country industrial clusters", Discussion Papers Series №103, ILO.

OECD (1999). "Managing National Innovation Systems". Paris OECD.

Porter, Michael (1990). "The Competitive Advantage of the Nations", The Free Press.

Porter, Michael. (1999a)."Cúmulos y Competencia. Nuevos objetivos para Empresas, Estados e Instituciones", en Ser competitivo. Nuevas aportaciones y conclusiones, Deusto, Bilbao.

Porter, Michael (1999b). "Los clusters y la competencia" Harvard Business Review, Vol. 1 №2.

Prahalad, C.K. (1999). "Los desafíos emergentes" Trend Management/ResearchTechnology Management, Vol. 1 №4 mayo-junio.

Puga, Diego y Venables, Anthony (1998). "Trading Arrangements and Industrial De- velopment". The World Bank Economic Review, Vol. 12, №2.

Ramos, Joseph (1998). "Una estrategia de desarrollo a partir de complejos productivos en torno a los recursos naturales", Revista CEPAL, № 66.

Roelandt, T. J. A. y Den Hertog, Pim (1998). "Cluster Analysis and Cluster-based Policy in OECD-countries. Various approaches, early results and policy implications" Draft synthesis report on phase 1, OECD-Focus Group on industrial clusters, The Hague/Utrecht.

Schmitz, Hubert (1990). "Small firms and flexible specialisation in developing countries". Labour and Society, 15 (3).

Schmitz, Hubert (1995). "Collective Efficiency: Growth for Small-Scale Industry". Journal of Development Studies, 34.

Schmitz, Hubert (1997). "Collective Efficiency and increasing returns", IDS working paper 50, University de Sussex, Reino Unido.

Selznik, Philip (1957). "Leadership in Administration", Nueva York, Harper \& Row.

Sutz, Judith (2000). "The University-IndustryGovernment Relations in Latin America”. Research Policy, № 29.

Venables, Anthony (1993). "Equilibrium locations of vertically linked industries". CEPR. Discussion Paper Series, No. 802.

Venables, Anthony (1996). "Localization of industry and trade performance". Oxford University Press Oxford Review of Economic Policy.

Weber, Alfred (1929). "Theory of the Location of Industries". Trans. C.J. Friedrich. Chicago: University of Chicago Press.

Weller, Jürgen (2000). "Reformas económicas, crecimiento y empleo: Los mercados de trabajo en América Latina y el Caribe", Centro de Estudios para América Latina y el Caribe (CEPAL), Santiago de Chile. 